Supporting Information

\title{
Sponge Graphene Aerogel Pressure Sensors with Extremely-Wide Operation Range for Human Recognition and Motion Detection
}

Ziyu Yue $e^{1}$, Yucan Zhu', Jianxing Xial ${ }^{1}$, Yi Wang ${ }^{1}$, Xingke Ye $e^{1}$,

Hedong Jiang ${ }^{1}$, Hongbo Jia ${ }^{2}$, Yuan Lin ${ }^{1}$ \& Chunyang Jia ${ }^{1 *}$

${ }^{1}$ State key Laboratory of Electronic Thin Films and Integrated Devices, School of

Electronic Science and Engineering, ${ }^{2}$ Glasgow College, University of Electronic

Science and Technology of China, Chengdu 610054, P. R. China

${ }^{*}$ Corresponding author. Tel.:+86 28 83201991; Fax: +86 28 83202569; Email: cyjia@uestc.edu.cn (C.Y.Jia) 


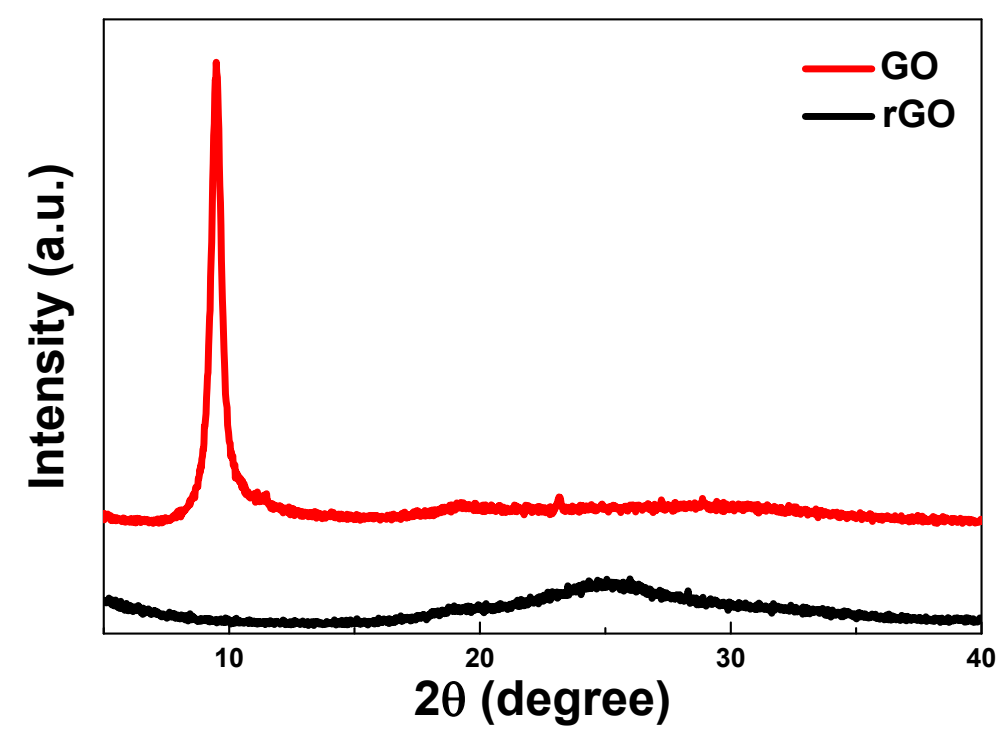

Figure S1. XRD patterns of GO and rGO.

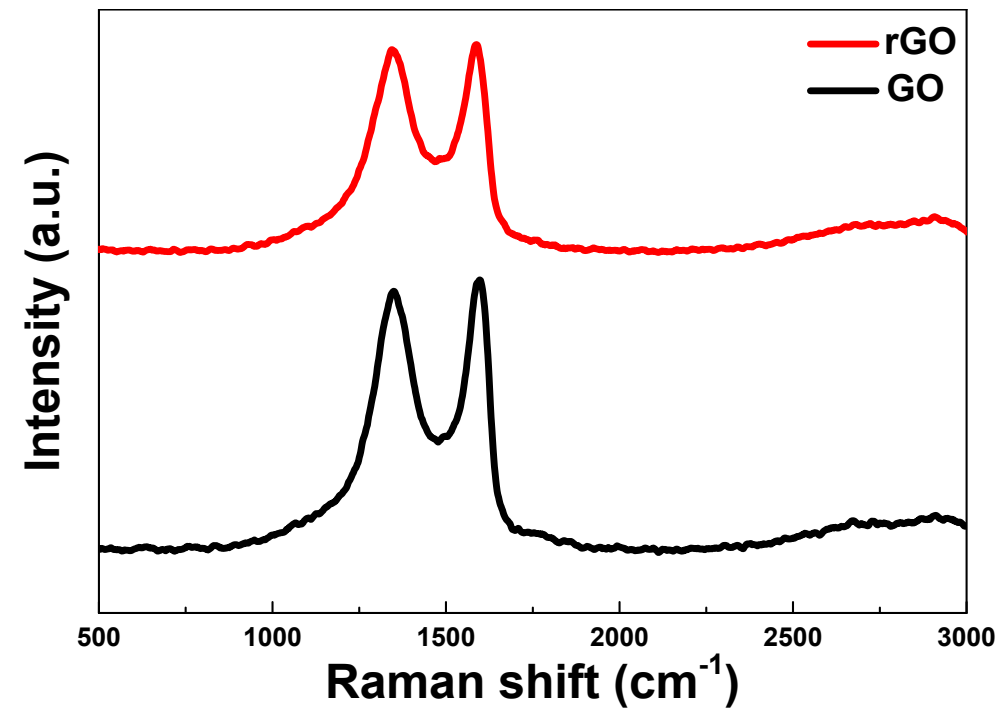

Figure S2. Raman results of GO and rGO. 


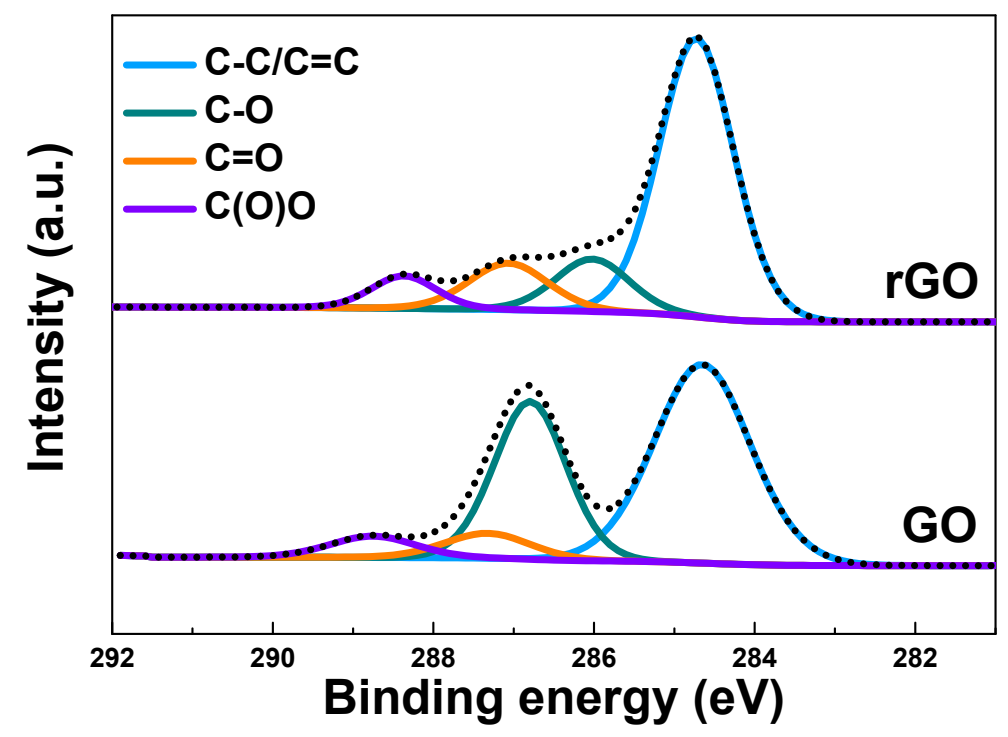

Figure S3. XPS results of GO and rGO.

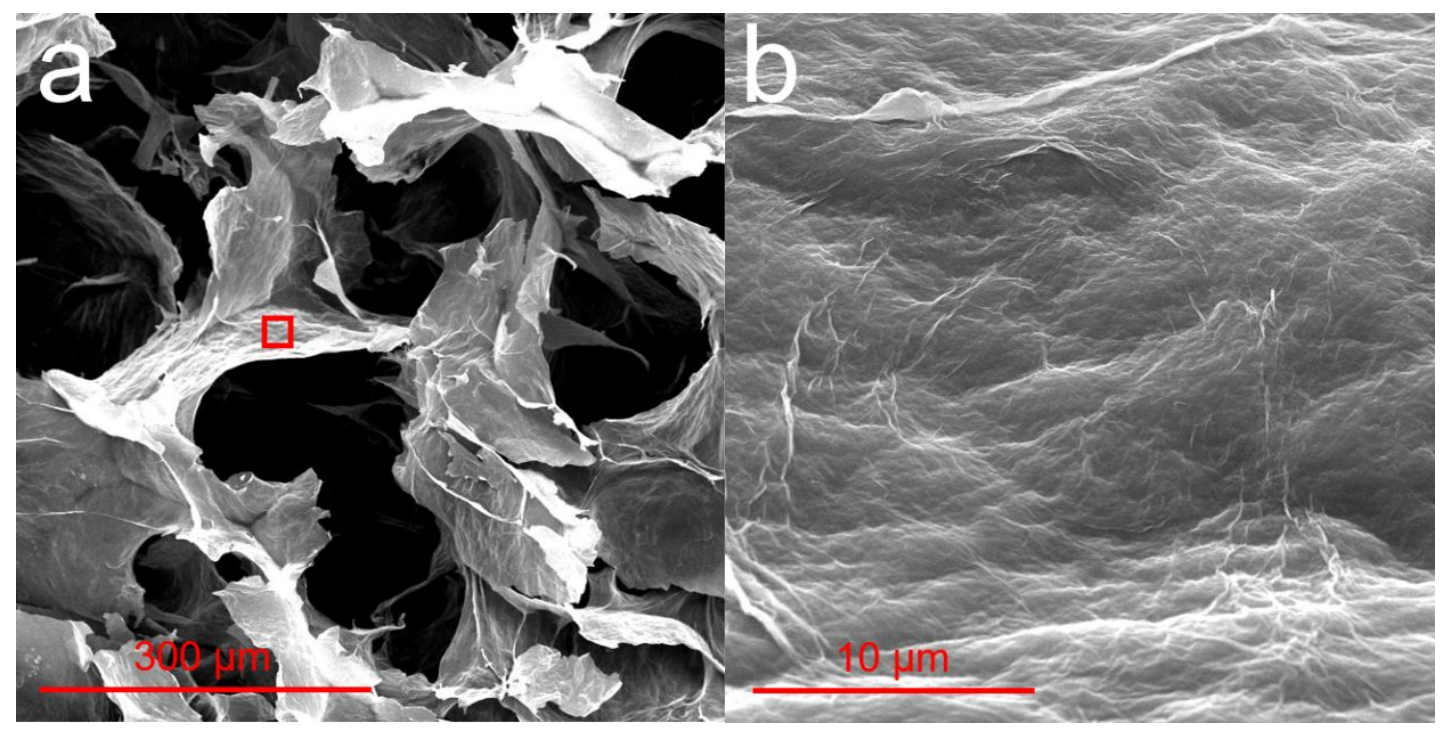

Figure S4. SEM images (a) and high-resolution image (b) of lyophilizated Ca-alginate graphene hydrogel without glycerin decorated. 


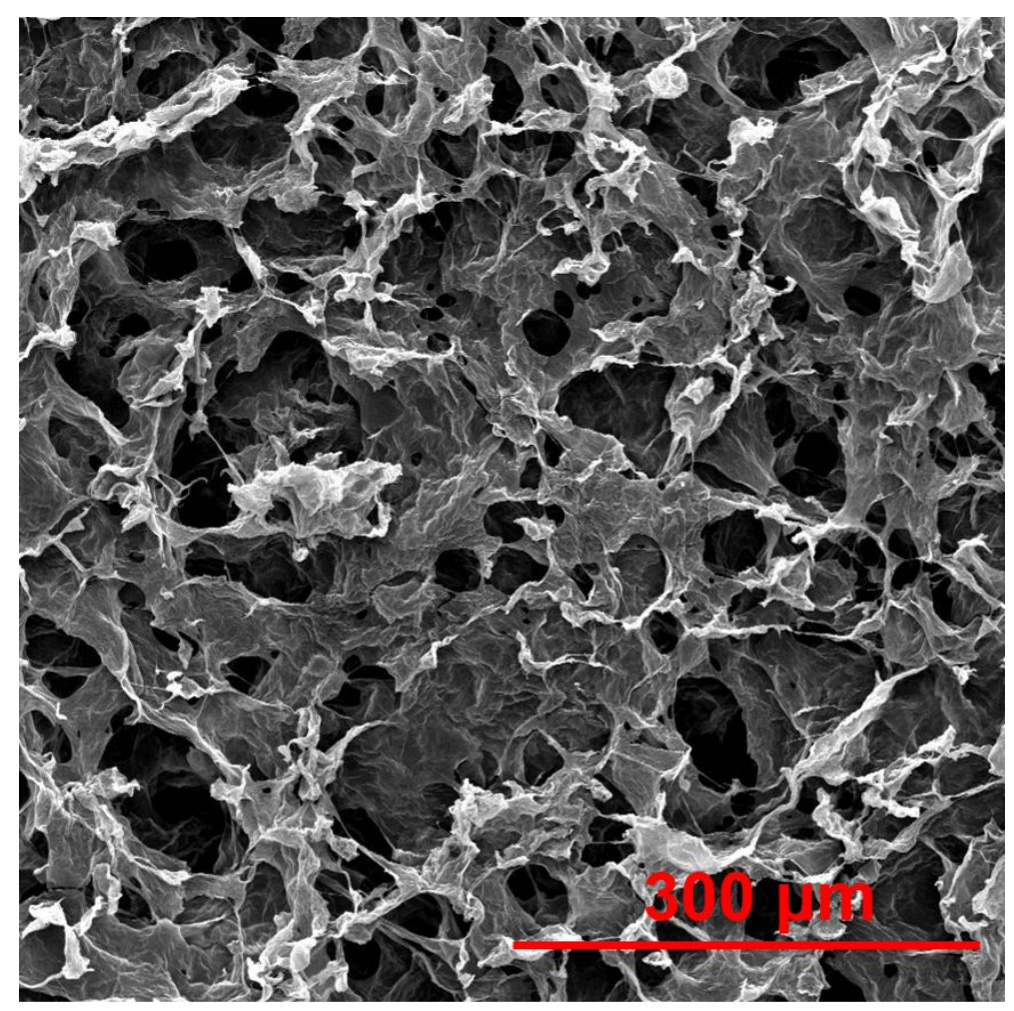

Figure S5. SEM image of 1:10 glycerin/water proportion of Calcium lactate solution sample.

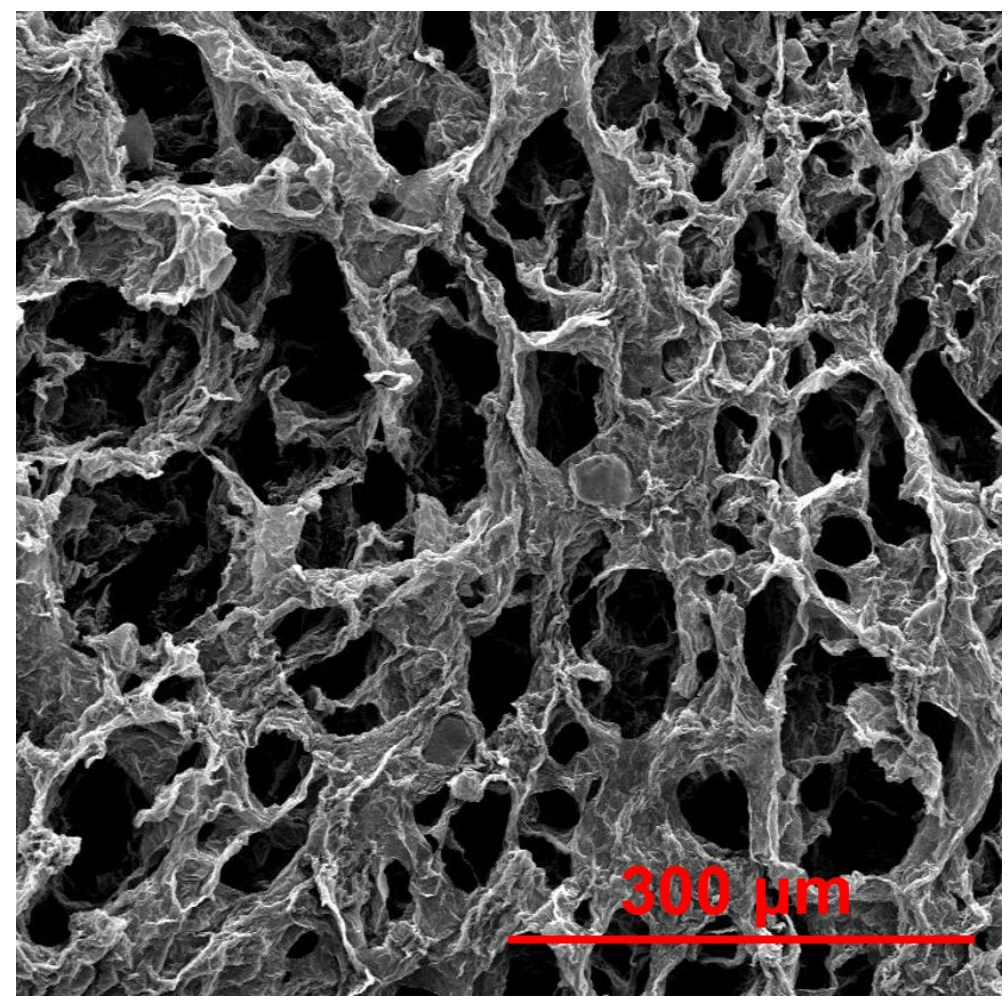

Figure S6. SEM image of 1:1 glycerin/water proportion of Calcium lactate solution sample. 


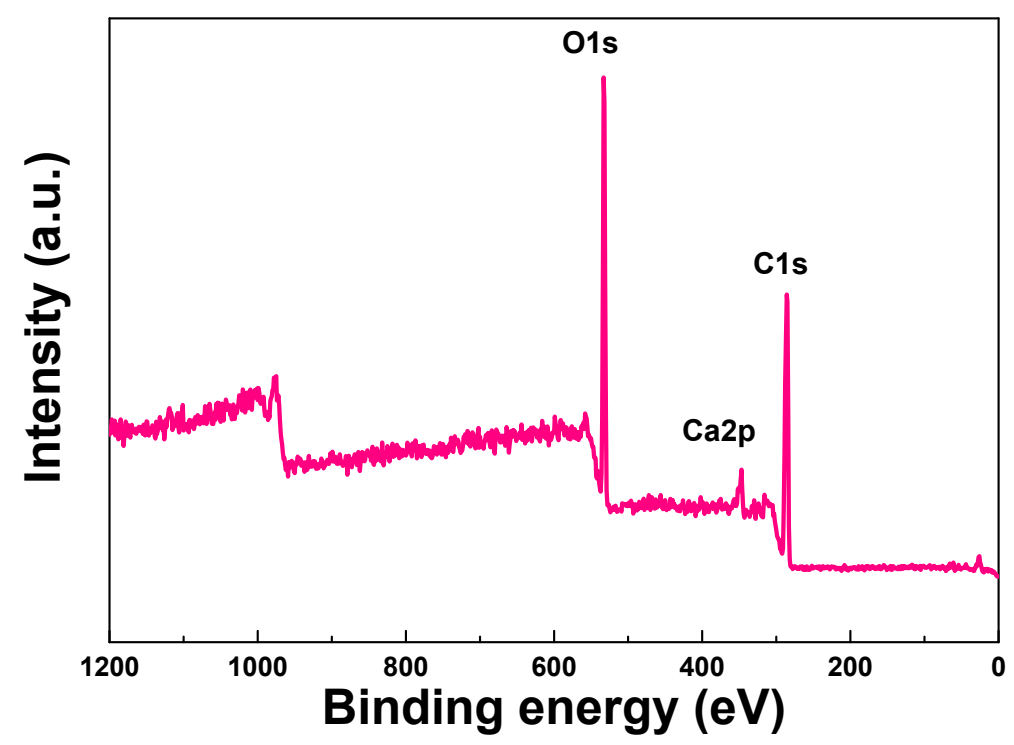

Figure S7. XPS survey pattern of SGA.

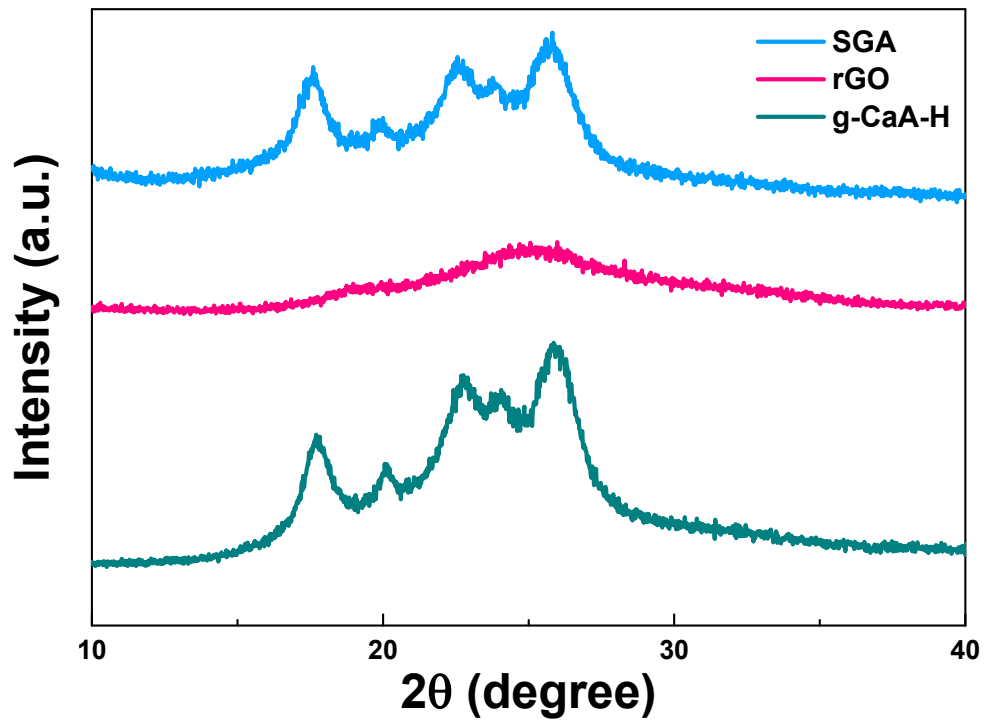

Figure S8. Raman results of GO and rGO. 


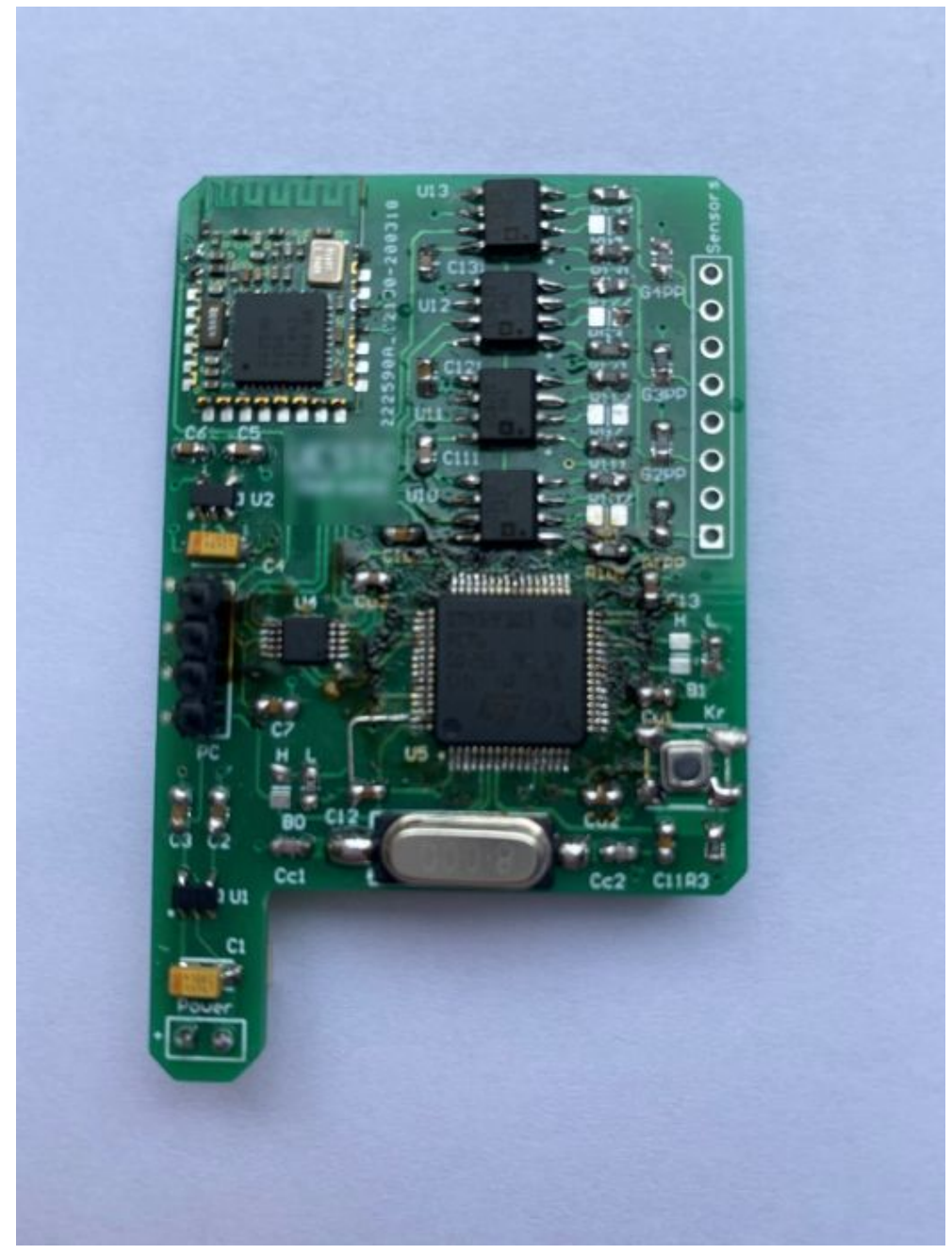

Figure S9. Image of controlling circuit board.

\begin{tabular}{llll}
\hline & Height & Weight & Foot size \\
\hline Tester 1 & $165 \mathrm{~cm}$ & $58 \mathrm{~kg}$ & $25.5 \mathrm{~cm}$ \\
Tester 2 & $170 \mathrm{~cm}$ & $72 \mathrm{~kg}$ & $25.0 \mathrm{~cm}$ \\
Tester 3 & $174 \mathrm{~cm}$ & $65 \mathrm{~kg}$ & $26.0 \mathrm{~cm}$
\end{tabular}

Table S1. Basic information of three testers in height, weight and foot size. 


\begin{tabular}{lcccc}
\hline & Sensitivity & Operation range & Response time & Durability \\
\hline Ref. 1 & $2.49 \mathrm{kPa}^{-1}$ & $270 \mathrm{kPa}$ & $<50 \mathrm{~ms}$ & 200 \\
Ref. 2 & $0.35 \mathrm{kPa}^{-1}$ & $25 \mathrm{kPa}$ & $48 \mathrm{~ms}$ & 3000 \\
Ref. 3 & $0.55 \mathrm{kPa}^{-1}$ & $400 \mathrm{kPa}$ & $0.93 \mathrm{~s}$ & 3600 \\
Ref. 4 & $0.096 \mathrm{kPa}^{-1}$ & $175 \mathrm{kPa}$ & Not declared & 10000 \\
Ref. 5 & $39 \% \mathrm{kPa}^{-1}$ & $25 \mathrm{kPa}$ & $0.19 \mathrm{~s}$ & 6000 \\
Ref. 6 & $81.61 \mathrm{kPa}^{-1}$ & $100 \mathrm{kPa}$ & $6 \mathrm{~ms}$ & 800 \\
This work & $5 \mathrm{kPa}^{-1}$ & $1000 \mathrm{kPa}$ & $8 \mathrm{~ms}$ & 4000 \\
\hline
\end{tabular}

Table S2. Performance comparing among SGAPS and recently reported pressure sensors in Sensitivity, operation range response time and durability.

\section{References}

(1) Chen, W.; Wang, B.; Zhu, Q.; Yan, X., Flexible Pressure Sensors with a Wide Detection Range Based on Self-Assembled Polystyrene Microspheres. Sensors 2019, $19,5194$.

(2) Guo, Y.; Gao, S.; Yue, W.; Zhang, C.; Li, Y., Anodized Aluminum OxideAssisted Low-Cost Flexible Capacitive Pressure Sensors Based on Double-Sided Nanopillars by a Facile Fabrication Method. ACS Appl. Mater. Interfaces 2019, 11, 48594-48603.

(3) Zhao, J.; Fu, Y.; Xiao, Y.; Dong, Y.; Wang, X.; Lin, L., A Naturally Integrated Smart Textile for Wearable Electronics Applications. Adv. Mater. Technol. 2019, 5, 1900781.

(4) Tang, Z.; Jia, S.; Zhou, C.; Li, B., 3D Printing of Highly Sensitive and LargeMeasurement-Range Flexible Pressure Sensors with a Positive Piezoresistive Effect. ACS Appl. Mater. Interfaces 2020, 12, 28669-28680. 
(5) Zhang, Y.; Liu, S.; Miao, Y.; Yang, H.; Chen, X.; Xiao, X.; Jiang, Z.; Chen, X.; Nie, B.; Liu, J., Highly Stretchable and Sensitive Pressure Sensor Array Based on Icicle-Shaped Liquid Metal Film Electrodes. ACS Appl. Mater. Interfaces 2020, 12, 27961-27970.

(6) Liu, K.; Yu, J.; Li, Y.; Yan, X.; Bai, D.; Liao, X.; Zhou, Z.; Gao, Y.; Yang, X.;

Li, L., Carbon Black from Diesel Soot for High-Performance Wearable Pressure Sensors. Adv. Mater. Technol. 2019, 4, 1900475. 